\title{
Exposure to Chinese famine in early life and the risk of sensory impairment in adulthood
}

\author{
Hongguo Rong, ${ }^{1,2}$ Xiaozhen Lai, ${ }^{1}$ Elham Mahmoudi, ${ }^{3}$ Hai Fang (D) 1,4,5
}

\begin{abstract}
${ }^{1}$ China Center for Health Development Studies, Peking University, Beijing, China ${ }^{2}$ Institute for Excellence in Evidence-Based Chinese Medicine, Beijing University of Chinese Medicine, Beijing, China

${ }^{3}$ Department of Family Medicine, University of Michigan, Ann Arbor, Michigan USA

${ }^{4}$ Peking University Health Science Center-Chinese Center for Disease Control and Prevention Joint Center for Vaccine Economics, Beijing, China

${ }^{5}$ Key Laboratory of Reproductive Health, National Health Commission of the People's Republic of China, Beijing, China.
\end{abstract}

Correspondence to Hai Fang, China Center for Health Development Studies, Peking University, Beijing 100083, China; hfang@hsc.pku.edu.cn

Received 13 January 2020 Revised 28 April 2020 Accepted 31 July 2020 Published Online First 28 August 2020

Check for updates

(c) Author(s) (or their employer(s)) 2021. No commercial re-use. See rights and permissions. Published by BMJ.

To cite: Rong $\mathrm{H}$, Lai $\mathrm{X}$

Mahmoudi E, et al. J

Epidemiol Community Health 2021:75:16-21.

\section{ABSTRACT}

Background Studies have shown that malnutrition in early life has a negative effect on midlife cognitive functions. Little is known, however, about the relationship between early-life malnutrition and visual, hearing or dual sensory impairments in adulthood. This study aims to investigate the association between exposure to the 1959-1961 Chinese famine in early life and sensory impairments in adulthood.

Methods A total of 6347 adults born between 1952 and 1964 surveyed in the 2015 China Health and Retirement Longitudinal Study were included in this study. The presence of sensory impairments was identified by self-reported assessment of visual and hearing functions. The associations between multi-stage early-life famine exposure and sensory functions were estimated using the multiple generalised linear model.

Results Compared with the unexposed group, respondents exposed to famine in the fetal period and late childhood had a significantly higher risk of hearing impairment (OR 1.54, 95\% Cl 1.06 to 2.24; OR 1.75, $95 \% \mathrm{Cl} 1.23$ to 2.50 ) and dual sensory impairments (OR $1.45,95 \% \mathrm{Cl} 1.00$ to $2.11 ; \mathrm{OR} 1.55,95 \% \mathrm{Cl} 1.09$ to 2.21 ), respectively. Furthermore, in severely famineaffected areas, the early, mid and late childhood-exposed groups were more likely to have hearing and dual sensory impairments than the unexposed group. Those associations were not observed in less famine-affected areas. However, no significant association was found between famine exposure and visual impairment in earlylife famine-exposed group.

Conclusion Exposure to Chinese famine in the fetal period and late childhood was linked to hearing and dual sensory impairments in adulthood.

\section{INTRODUCTION}

Visual, hearing and dual sensory impairments are common among older adults ${ }^{1}$ and have been consistently associated with greater risk of cognitive decline, depression symptoms and reduction in quality of life. ${ }^{2}$ Intrauterine nutrition may play an important role in the development of sensory systems. For instance, a cross-sectional observational study that sampled 433390 individuals in the UK suggests that being underweight at birth is a risk factor for poorer vision, hearing and cognition in midlife. ${ }^{4}$ As a major health problem, malnutrition affects millions of children across the world, especially in low- and middle-income countries. ${ }^{5}$

Historical famines provide a unique opportunity to study the long-term association between nutritional deprivation in early life and age-related visual and hearing impairments. As one of the largest catastrophic events in recent human history, the
1959-1961 Chinese famine caused approximately 30 million excess deaths. ${ }^{6}$ In contrast to the 1944-1945 Dutch famine and 1932-1933 Holodomor famine, Chinese famine lasted for 3 years and influenced almost every region of China. ${ }^{6}$ The 1959-1961 Chinese famine allows researchers to investigate the long-term influence of malnutrition in intrauterine and childhood periods among the non-European population.

A previous study has found that pregnancy and infancy malnutrition played an important role in later disease susceptibility. ${ }^{7}$ Deficits in sensory functions like vision and hearing impairments may be a risk factor for the development of Alzheimer's disease (AD) and other late-life degenerative conditions. ${ }^{8}$ However, the association between severe nutritional deficiency during early life and sensory impairments remains to be investigated. Thus, it is salient to examine the associations between severe nutritional deprivation during early life and visual, hearing, and dual sensory impairments (visual and hearing) in adulthood. In this study, we used data from the 2015 China Health and Retirement Longitudinal Study (CHARLS) to examine this topic.

\section{METHODS}

\section{Study population}

Data from the 2015 CHARLS were used to investigate the association between fetal/childhood famine exposure and adult sensory functions. CHARLS is an ongoing national longitudinal study of Chinese adults aged 45 years and older, which collects an excellent nationwide sample to represent the middle-aged and elderly in China. Its rationale and cohort design has previously been described in detail. $^{9}$ The 2015 wave is the latest follow-up survey, which sampled 21095 respondents, and all of them signed informed consent. The programme protocol was in line with the Declaration of Helsinki and was ethically approved by the Biomedical Ethics Review Committee of Peking University (approval number: IRB00001052$11015) .{ }^{10}$

\section{Famine cohorts and area categories}

Figure 1 shows the schematic flow of sample selection. A total of 6347 samples were finally included based on the following inclusion and exclusion criteria. Inclusion criteria were as follows: born between 1952 and 1964; having the ability to communicate and recall clearly. Exclusion criteria were as follows: legally blind; suffering from severe memory-related diseases (such as $\mathrm{AD}$, brain atrophy, 


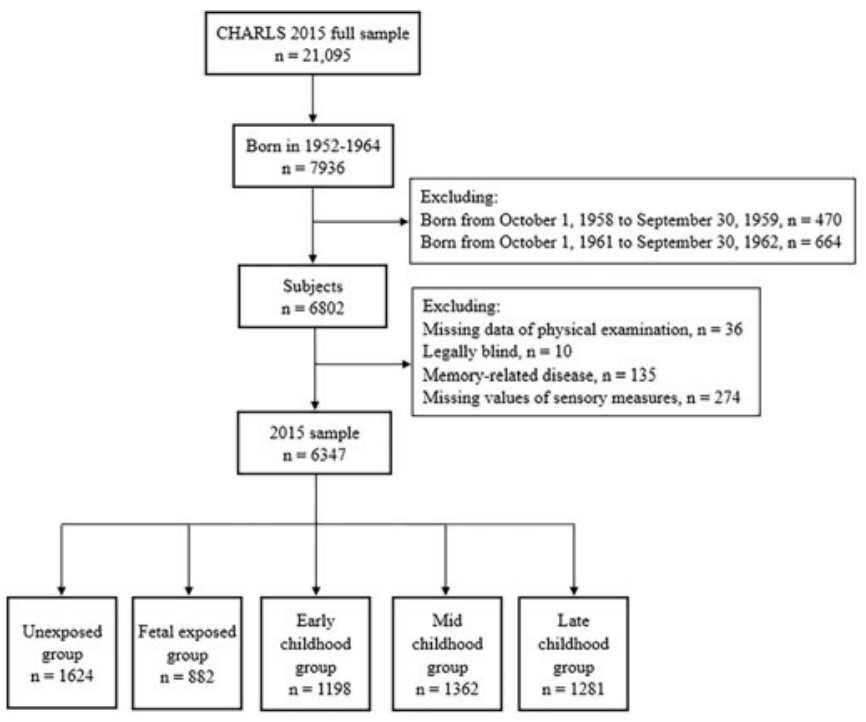

Figure 1 Flow chart of sample selection from 2015 China Health and Retirement Longitudinal Study (CHARLS) survey.

Parkinson's disease); having missing values of physical examination and sensory measures. In line with previous studies on Chinese famine, respondents were categorised into five groups based on their date of birth: the unexposed group (1 October 1962-30 September 1964), the fetal-exposed group (1 October 1959-30 September 1961), the early childhood-exposed group (1 October 1956-30 September 1958), the mid childhood-exposed group (1 October 1954-30 September 1956) and the late childhood-exposed group (1 October 1952-30 September 1954).

Due to undefinable starting and ending dates of the Chinese famine, respondents born between 1 October 1958 and 30 September 1959, and between 1 October 1961 and 30 September 1962 were excluded to minimise misclassification. Following the method used in the previous Chinese famine study, ${ }^{11}$ excess death rate (EDR) of each province was used as a proxy for famine severity. Taking $100 \%$ EDR as the threshold, severity of famine-affected areas was determined at the provincial level, so as to distinguish the respondents in each of the five exposure cohorts into severely or less- to severely famineaffected populations.

\section{Assessment of sensory functions}

Similar to the Survey of Health in the USA, the presence of sensory impairments in CHARLS was measured using selfreported assessment of visual and hearing functions. Visual function was assessed using two questions: 'How good is your eyesight for seeing things at a distance, like recognising a friend from across the street (with glasses or corrective lenses if you wear them)? Would you say your eyesight for seeing things at a distance is excellent (1), very good (2), good (3), fair (4) or poor (5)?' and 'How good is your eyesight for seeing things up close, like reading ordinary newspaper print (with glasses or corrective lenses if you wear them)? Would you say your eyesight for seeing things up close is excellent (1), very good (2), good (3), fair (4) or poor (5)?'. Hearing function was evaluated using the question: 'Is your hearing very good, good, fair, poor or very poor (with a hearing aid if you normally use it and without if you normally don't)? Would you say your hearing is excellent (1), very good (2), good (3), fair (4) or poor (5)?'. Based on the answers to the three questions, we categorised respondents as having visual or hearing impairment if they reported having fair or poor vision (for either distance or near vision) or hearing function. ${ }^{12}$ In line with the Health and Retirement Study Cohort study, ${ }^{13}$ we defined four states of sensory impairments as follows: no impairment, visual impairment but no hearing impairment, hearing impairment but no visual impairment and dual sensory impairments.

\section{Assessment of covariates}

Respondents' sociodemographic characteristics, health behaviours and health conditions were measured in CHARLS faceto-face interviews by trained interviewers. The covariates included age, gender, education level, smoking status, alcohol consumption status, marital status (married and cohabitation, or otherwise), Hukou status (urban or rural), self-reported general health status, depressive symptoms and chronic disease conditions. Education level was categorised as 'illiterate', 'primary or middle school' and 'high school and above'. Regarding selfreported health status, including both current conditions and status in childhood, 'excellent', 'very good' and 'good' were defined as 'good', whereas 'fair' and 'poor' were defined as 'poor'. Depressive symptoms were estimated by Center for Epidemiologic Studies Depression Scale-10 score, which indicates clinically significant depressive symptoms. ${ }^{14}$ Chronic disease conditions were detected using the presence of chronic diseases based on self-reported doctor-diagnosed diseases, including hypertension, diabetes, dyslipidemia, stroke, kidney disease, asthma, chronic lung diseases, liver disease, stomach diseases and heart problems.

\section{Statistical analysis}

In the present study, continuous variables with non-normal distribution were reported as medians (IQRs), and discrete variables were presented as percentages (\%). We performed Kruskal-Wallis test to compare the difference in skewed parameters and $?^{2}$ test or Fisher's exact test to compare the frequencies of categorical variables. The Dunnett corrections were adopted to perform post hoc comparisons with the unexposed group.

The generalised linear model (GLM) was used to estimate the association between fetal period and multi-stage childhood famine exposure and the risk of visual, hearing and dual sensory impairments in adulthood. The unexposed group was considered as the reference. Age, gender, education, famine severity, smoking, drinking, depressive symptoms, self-reported current health conditions, health status in childhood, hypertension, diabetes, chronic lung disease and heart diseases were included as control variables in the analyses. In view of the spouse data collected in CHARLS, we corrected the family cluster by adding a cluster (household) in the GLM. In addition, we conducted stratified analyses according to famine severity to distinguish severely and less severely famine-affected areas more appropriately. The interaction between famine-affected areas and different life stages of famine exposure was tested using a multiplicative factor in the logistic regression model. GLM results were reported as ORs with 95\% CIs. All statistical analyses were conducted using STATA, version 13.0 (StataCorp., College Station, TX, USA).

\section{RESULTS}

Table 1 shows the demographic and clinical characteristics of participants in this study. In total, 3268 females and 3079 males were included. Of the total study sample $(n=6347), 882(13.9 \%)$ participants were exposed to the famine during the fetal period, and 3841 (60.5\%) were exposed during childhood. Compared 
Table 1 General characteristics of study population by Chinese famine exposure

\begin{tabular}{|c|c|c|c|c|c|}
\hline & & & Childhood-expo & & \\
\hline & Unexposed & Fetal-exposed & Early childhood & Mid childhood & Late childhood \\
\hline $\mathrm{N}$ & 1624 & 882 & 1198 & 1362 & 1281 \\
\hline Birth date* & 1962-1964 & 1959-1961 & 1956-1958 & 1954-1956 & 1952-1954 \\
\hline Age in 2015 & $52.0(1)$ & $55.1(1) \dagger$ & $58.0(1) \dagger$ & $60.1(1) \dagger$ & $62.0(2) \dagger$ \\
\hline Male, $n(\%)$ & $791(48.7)$ & $409(46.4)$ & $597(49.8)$ & $666(48.9)$ & $616(48.1)$ \\
\hline Severely famine-affected areas, $\mathrm{n}(\%)$ & - & $284(32.2)$ & $442(36.9)$ & $544(39.9)$ & $515(40.2)$ \\
\hline Education, n (\%) & & & & & \\
\hline Illiterate & $163(10.1)$ & $135(15.3)$ & $285(23.8)$ & $355(26.1)$ & $387(30.2)$ \\
\hline Primary or middle school & $1161(71.6)$ & $529(60.0)$ & $699(58.4)$ & $828(60.8)$ & $780(60.9)$ \\
\hline High school and above & $298(18.4)$ & $218(24.7)$ & $213(17.8)$ & $178(13.1)$ & $114(8.9)$ \\
\hline Smoking, n (\%) & $464(28.6)$ & $257(29.1)$ & $365(30.5)$ & $427(31.4)$ & $382(29.8)$ \\
\hline Drinking, n (\%) & $638(39.3)$ & $340(38.6)$ & $465(38.9)$ & $488(35.9)$ & $435(34.0) \dagger$ \\
\hline Marital status & $1425(87.8)$ & $770(87.3)$ & $1035(86.4)$ & $1166(85.6)$ & $1091(85.2)$ \\
\hline Hukou status, n (\%) & & & & & \\
\hline Urban & $344(22.3)$ & $195(23.4)$ & $258(22.5)$ & $274(21.3)$ & $257(21.3)$ \\
\hline Rural & $1200(77.7)$ & $639(76.6)$ & $877(77.5)$ & $1013(78.7)$ & $948(78.7)$ \\
\hline Self-reported poor health status, $\mathrm{n}(\%)$ & $1155(71.3)$ & $640(72.3)$ & $893(74.5)$ & $1019(74.9)$ & $1001(78.2) \dagger$ \\
\hline Poor health status in childhood (self-reported), $n(\%)$ & $450(28.0)$ & $228(26.2)$ & $344(29.1)$ & $419(31.1)$ & $394(31.3)$ \\
\hline Depression, n (\%) & $498(30.8)$ & $252(28.6)$ & $387(32.4)$ & $433(32.0)$ & $457(35.8) \dagger$ \\
\hline Hypertension, $\mathrm{n}(\%)$ & $387(26.1)$ & $241(30.4)$ & $334(30.4)$ & $397(31.5) \dagger$ & $440(36.9) \dagger$ \\
\hline Diabetes, n (\%) & $221(14.3)$ & $157(18.5) \dagger$ & $204(17.6)$ & $244(18.5) \dagger$ & $246(19.9) \dagger$ \\
\hline Dyslipidemia, $\mathrm{n}(\%)$ & $262(18.1)$ & $169(22.8)$ & $217(20.2)$ & $245(19.7)$ & $241(20.9)$ \\
\hline Stroke, n (\%) & $34(2.3)$ & $20(2.5)$ & $24(2.1)$ & $34(2.7)$ & $41(3.5)$ \\
\hline Kidney disease, $\mathrm{n}(\%)$ & $136(9.2)$ & $84(10.6)$ & $129(11.9)$ & $144(11.5)$ & $126(10.7)$ \\
\hline Asthma, $\mathrm{n}(\%)$ & $55(3.7)$ & $34(4.3)$ & $55(5.0)$ & $65(5.2)$ & $67(5.6)$ \\
\hline Chronic lung diseases, n (\%) & $160(10.9)$ & $98(12.3)$ & $154(14.1)$ & $164(13.0)$ & $180(15.3) \dagger$ \\
\hline Liver disease, n (\%) & $100(6.8)$ & $62(7.9)$ & $81(7.5)$ & $93(7.4)$ & $79(6.7)$ \\
\hline Stomach disease, n (\%) & $494(33.2)$ & $264(33.4)$ & $368(33.6)$ & $429(34.1)$ & $390(32.8)$ \\
\hline Heart diseases, n (\%) & $188(12.8)$ & $119(15.0)$ & $191(17.6) \dagger$ & $217(17.2) \dagger$ & $231(19.5) \dagger$ \\
\hline
\end{tabular}

*From 1 October year to 30 September year. Continuous variables are expressed as median (IQR), and categorical variables are shown as $n$ (\%). The Kruskal-Wallis test was used for continuous variables with a skewed distribution, and the Pearson's $\chi^{2}$ test for categorical variables when comparing differences.

$\mathrm{tp}<0.05$ (after Dunnett corrections).

with unexposed individuals, participants in early life-exposed groups had lower education levels, and those in childhoodexposed groups had higher prevalence of hypertension, diabetes and heart diseases. Moreover, late childhood-exposed individuals were less likely to drink, and more likely to report poor health status, have chronic lung diseases and have depressive symptoms. Meanwhile, the prevalence rate of diabetes in the fetal-exposed group was higher than that in the unexposed group after Dunnett corrections.

Table 2 provides the results of sensory function measures in five famine-exposed groups. After Dunnett corrections, participants in mid and late childhood-exposed groups had higher prevalence of self-reported hearing impairment and dual sensory impairments than those in the unexposed group. Compared with the unexposed group, while the prevalence of selfreported visual impairment was higher in fetal- and childhoodexposed groups, significance was not shown after Dunnett corrections.

Table 3 indicates the relationship between famine exposure and subsequent self-reported visual and/or hearing impairment in multiple GLM. Compared with the unexposed group, the participants of late childhood-exposed group had higher risk of visual impairment, hearing impairment and dual

\begin{tabular}{|c|c|c|c|c|c|}
\hline & \multirow[b]{2}{*}{ Unexposed } & \multirow[b]{2}{*}{$\begin{array}{l}\text { Fetal- } \\
\text { exposed }\end{array}$} & \multicolumn{3}{|c|}{ Childhood-exposed } \\
\hline & & & $\begin{array}{l}\text { Early } \\
\text { childhood }\end{array}$ & $\begin{array}{l}\text { Mid } \\
\text { childhood }\end{array}$ & $\begin{array}{l}\text { Late } \\
\text { childhood }\end{array}$ \\
\hline $\begin{array}{l}\text { Visual } \\
\text { impairment }\end{array}$ & $1331(82.0)$ & 730 (82.8) & $995(83.1)$ & $1136(83.4)$ & $1063(83.0)$ \\
\hline $\begin{array}{l}\text { Hearing } \\
\text { impairment }\end{array}$ & 964 (59.4) & $527(59.8)$ & $760(63.4)$ & $890(65.4)^{*}$ & $862(67.3)^{*}$ \\
\hline $\begin{array}{l}\text { Dual sensory } \\
\text { impairments }\end{array}$ & $897(55.2)$ & $492(55.8)$ & $700(58.4)$ & $830(60.9)^{*}$ & $787(61.4)^{*}$ \\
\hline
\end{tabular}

${ }^{*} \mathrm{p}<0.05$ (after Dunnett corrections).

Data are presented as $n(\%)$. The Pearson's $\chi^{2}$ test for categorical variables when comparing differences.

sensory impairments, after adjusting for age and gender. Meanwhile, the mid childhood-exposed group had a risk of hearing impairment. Further adjusted for other control variables, the famine-sensory function association disappeared between both late childhood exposure and dual sensory impairments, and mid childhood exposure and hearing impairment. Interestingly, participants exposed to the famine 
Table 3 Multiple GLM estimates of the association between famine exposure and sensory impairments in 2015

\begin{tabular}{|c|c|c|c|c|c|}
\hline & \multirow[b]{2}{*}{ Unexposed } & \multirow[b]{2}{*}{ Fetal-exposed } & \multicolumn{3}{|l|}{ Childhood-exposed } \\
\hline & & & Early childhood & Mid childhood & Late childhood \\
\hline \multicolumn{6}{|l|}{ Visual impairment } \\
\hline OR $(95 \% \mathrm{Cl})^{*}$ & 1.00 (ref.) & $1.26(0.84$ to 1.89$)$ & 1.46 (0.73 to 2.94$)$ & 1.30 (0.75 to 2.23$)$ & $1.50(1.02$ to 2.21$)$ \\
\hline OR $(95 \% \mathrm{Cl}) \dagger$ & 1.00 (ref.) & 1.29 (0.80 to 2.06$)$ & 1.18 (0.53 to 2.644$)$ & 0.87 (0.47 to 1.61$)$ & 1.12 (0.72 to 1.75$)$ \\
\hline \multicolumn{6}{|c|}{ Hearing impairment } \\
\hline OR $(95 \% \mathrm{Cl})^{*}$ & 1.00 (ref.) & $1.24(0.90$ to 1.70$)$ & 1.50 (0.86 to 2.61 ) & 1.69 (1.10 to 2.59$)$ & 1.74 (1.28 to 2.37$)$ \\
\hline OR $(95 \% \mathrm{Cl}) \dagger$ & 1.00 (ref.) & 1.54 (1.06 to 2.24$)$ & 1.34 (0.71 to 2.53 ) & 1.57 (0.95 to 2.58 ) & 1.75 (1.23 to 2.50$)$ \\
\hline \multicolumn{6}{|c|}{ Dual sensory impairments } \\
\hline OR $(95 \% \mathrm{Cl})^{*}$ & 1.00 (ref.) & 1.21 (0.89 to 1.66$)$ & 1.37 (0.80 to 2.36$)$ & 1.47 (0.97 to 2.25$)$ & 1.59 (1.18 to 2.15$)$ \\
\hline OR $(95 \% \mathrm{Cl}) \dagger$ & 1.00 (ref.) & 1.45 (1.00 to 2.11$)$ & 1.22 (0.68 to 2.37$)$ & 1.32 (0.80 to 2.17$)$ & 1.55 (1.09 to 2.21$)$ \\
\hline
\end{tabular}

*Adjusted for age and gender.

†Adjusted for the age, gender, education, famine severity, smoking, drinking, depressive symptoms, self-reported current health conditions, health status in childhood, hypertension, diabetes, chronic lung disease and heart diseases.

GLM, generalised linear model; ref., reference group.

during the fetal period had a significantly higher risk of hearing impairment (OR 1.54, 95\% CI 1.06 to 2.24 ) and dual sensory impairments (OR 1.45, 95\% CI 1.00 to 2.11) than the unexposed group. The risk of hearing and dual sensory impairments for late childhood-exposed participants was 1.75 (95\% CI 1.23 to 2.50 ) and 1.59 (95\% CI 1.09 to 2.21), respectively. However, the association between self-reported visual impairment and famine exposure was not observed in the fully adjusted model.

In order to control the potential effect of age on the association between famine exposure and sensory impairment risk, we further conducted stratified analyses by famine severity (table 4). In severely famine-affected areas, the early, mid and late childhoodexposed groups had higher risk of hearing and dual sensory impairments. No significant association was observed between hearing or dual sensory impairments and famine exposure in less severely famine-affected areas, and visual impairment was not found to be related with famine exposure in both severely and less severely famine-affected areas. In addition, no significant interactions were found between famine severity and different life stages of famine exposure on prevalence risk of visual, hearing and dual sensory impairments.

\section{DISCUSSION}

Using a nationally representative sample of 6347 communitydwelling Chinese adults, our study underlined that famine exposure during the fetal period, and childhood was associated with hearing and dual sensory impairments in adulthood.

This study provides new evidence to the 'developmental origins of health and disease' (DOHaD) theory that early life stages may be crucial time windows for adult diseases. ${ }^{15}$ Recent famine

Table 4 Association between famine exposure and sensory impairments stratified by famine severity

\begin{tabular}{|c|c|c|c|c|c|}
\hline & \multirow[b]{2}{*}{ Unexposed } & \multirow[b]{2}{*}{ Fetal-exposed } & \multicolumn{3}{|l|}{ Childhood-exposed } \\
\hline & & & Early childhood & Mid childhood & Late childhood \\
\hline \multicolumn{6}{|l|}{ Visual impairment } \\
\hline \multicolumn{6}{|l|}{ Severely famine-affected areas } \\
\hline OR $(95 \% \mathrm{Cl})$ & 1.00 (ref.) & 1.60 (0.67 to 3.83$)$ & 1.14 (0.32 to 4.11$)$ & 1.23 (0.44 to 3.45$)$ & 1.61 (0.78 to 3.32$)$ \\
\hline \multicolumn{6}{|l|}{ Less famine-affected areas } \\
\hline OR $(95 \% \mathrm{Cl})$ & 1.00 (ref.) & 1.13 (0.64 to 2.01 ) & 1.27 (0.45 to 3.56$)$ & 0.68 (0.31 to 1.50$)$ & $0.88(0.50$ to 1.56$)$ \\
\hline $\mathrm{P}$ value for interaction term & & 0.562 & 0.217 & 0.917 & 0.759 \\
\hline \multicolumn{6}{|l|}{ Hearing impairment } \\
\hline \multicolumn{6}{|l|}{ Severely famine-affected areas } \\
\hline OR $(95 \% \mathrm{Cl})$ & 1.00 (ref.) & 1.78 (0.96 to 3.27$)$ & 2.77 (1.05 to 7.33$)$ & 2.80 (1.29 to 6.12$)$ & 2.50 (1.47 to 4.31$)$ \\
\hline \multicolumn{6}{|l|}{ Less famine-affected areas } \\
\hline OR $(95 \% \mathrm{Cl})$ & 1.00 (ref.) & 1.34 (0.83 to 2.16$)$ & 0.80 (0.34 to 1.84$)$ & 1.04 (0.54 to 2.00$)$ & 1.38 (0.86 to 2.21$)$ \\
\hline$P$ value for interaction term & & 0.501 & 0.146 & 0.372 & 0.409 \\
\hline \multicolumn{6}{|l|}{ Dual sensory impairments } \\
\hline \multicolumn{6}{|l|}{ Severely famine-affected areas } \\
\hline OR $(95 \% \mathrm{Cl})$ & 1.00 (ref.) & 1.56 (0.86 to 2.82 ) & 2.75 (1.06 to 7.14$)$ & 2.72 (1.26 to 5.88$)$ & 2.17 (1.27 to 3.70$)$ \\
\hline \multicolumn{6}{|l|}{ Less famine-affected areas } \\
\hline OR $(95 \% \mathrm{Cl})$ & 1.00 (ref.) & 1.32 (0.82 to 2.14 ) & 0.72 (0.31 to 1.66$)$ & 0.79 (0.41 to 1.52$)$ & $1.23(0.76$ to 1.97$)$ \\
\hline$P$ value for interaction term & & 0.703 & 0.236 & 0.363 & 0.471 \\
\hline
\end{tabular}

Interaction term: interaction between famine severity and different life stages of famine exposure. Model adjusted for age, gender, education, smoking, drinking, depressive symptoms, selfreported current health conditions, health status in childhood, hypertension, diabetes, chronic lung disease and heart diseases. ref., reference group. 
studies suggested that undernutrition during gestation could accelerate later-life cognitive decline and ageing, ${ }^{16}{ }^{17}$ and the reason may lie in affected brain size, brain perfusion parameters and premature brain ageing. ${ }^{18}$ Our previous study also suggested that early-life famine exposure might be associated with general cognition and visuospatial episodic memory in midlife. ${ }^{19}$ It was reported that hearing, visual and olfactory impairments are individually associated with cognition, and sensory impairment in midlife may be a marker of cognitive decline. ${ }^{20}$ However, there was limited evidence on the association between early-life malnutrition and adult sensorineural health. The United Kingdom Biobank study was the first to investigate the link between birth weight and late-life sensory problems, and suggested that abnormally large or small birth weight was significantly associated with poorer performance of cognitive and sensory function test. ${ }^{4}$ However, self-reported birth weight adopted in this study could potentially underestimate the association due to recall bias. Meanwhile, the everlasting adverse effects of prenatal malnutrition on late life were mainly due to underdeveloped organs and systems in early critical periods, instead of the altered body size at birth. ${ }^{21} 22$ In this study, we improved the generalisability of our findings by setting fetal and multi-stage childhood famineexposed cohorts.

Our findings revealed that exposure to Chinese famine during the prenatal period and late childhood increased the risk of hearing and dual sensory impairments in late life. Together with our previous findings, ${ }^{19} 23$ it was indicated that the starting point of hearing, vision and cognition impairments in midlife could date back to younger age. Our results showed that the prevalence of hearing and dual sensory impairments in the fetal-exposed group was higher than that in the unexposed group (although the difference was not statistically significant in table 2). We still found the associations between fetal famine exposure and hearing or dual sensory impairments after adjusting for control variables (table 3). Studies on the 1944-1945 Dutch famine have demonstrated that nutritional deprivation during prenatal period might increase nervous system anomalies at birth and thus cause irreversible brain damage. ${ }^{24}$ Malnutrition at that time might be associated with impaired brain functioning and neurotransmitter release. ${ }^{25}$ Although the interactions between famine severity and early-life famine exposure on the risk of visual, hearing and dual sensory impairments were not significant in the current study, the associations between early, mid and late childhood famine exposure and late-life hearing and dual sensory impairments were stronger among those exposed to severer famine. The results strongly support the 'first 1000 days' theory ${ }^{25}$ and indicated that fetus stage and childhood (age of $0-9$ years) are particularly vulnerable time periods of malnutrition reprogramming.

What attributes to this association between famine exposure and late-life sensory function decline? The Minnesota Starvation Experiment and 1944-1945 Dutch famine studies have shown that famine exposure may increase the susceptibility to chronic noncommunicable diseases, such as diabetes, dyslipidaemia and anaemia. ${ }^{26}{ }^{27}$ These chronic non-communicable diseases are recognised as dominant factors of cognitive and sensory function decline in midlife, with potential mechanism involving DNA methylation and cholesterol metabolism. ${ }^{28}$ The Genomic Research of the Chinese Famine study in China demonstrated that early-life exposure to severe famine could increase methylation in the insulin-like growth factor 2 gene, which would consequently increase cholesterol amount in midlife. ${ }^{29}$ Animal evidence proved that food restriction from weanling to 42 days of life among male Wistar rats could decrease glutamate release in the cortex, hippocampus, midbrain and brainstem at 370 days of life. ${ }^{30}$ It was indicated that malnutrition during critical periods of brain development caused an irreversible adverse effect on the auditory primary sensory pathway. ${ }^{30}$

The present study suggested that famine exposure in the fetal period and late childhood was positively associated with hearing and dual sensory impairments rather than visual impairment. Interestingly, although famine exposure was also reported to worsen visual-motor performance in late life, ${ }^{31}$ the association between famine exposure and risk of visual impairment did not reach statistical significance in the current study (though negative). This inconsistency may originate from different visual function assessment measures and different postnatal stages of famine exposure, and more detailed investigations can be conducted in the future. Meanwhile, it has been proven that the main causes of hearing impairment among Chinese elderly people are chronic diseases, presbycusis, long-term exposure to noise, otitis and media, ${ }^{32}$ and the occurrence of dual sensory impairments is positively associated with chronic diseases, depression, anxiety and cognitive impairment, especially for the elderly. ${ }^{12}{ }^{33}$ The above-mentioned reason could partly explain the phenomenon that famine-sensory function association was insignificant in terms of visual impairment but significant in terms of dual sensory impairments. However, we should be cautious about the generalisability of the main findings.

In the analysis of famine-sensory function associations, there exists potential bias caused by the use of hearing and vision measures, which allows for spectacles or hearing aids. The adoption of medical devices such as spectacles or hearing aids may make contributions to sensory rehabilitation, ${ }^{34}$ but in current times in China, only a small portion of adults with visual and/or hearing impairment use them to improve their sensory functions. The CHARLS 2013 data set suggested that for Chinese residents aged 60 years and above, only $10 \%$ visually impaired participants and $0.8 \%$ hearing impaired participants wore medical aids regularly. ${ }^{35}$ The use rate of aids was much lower than expected in China, suggesting that we could minimise the effects of aid use bias in the current study. ${ }^{36}$ Possible reasons for the low uptake of aids include financial constraints, misinformation and mistaken views (eg, spectacles may worsen vision loss, or hearing impairment is normal in the ageing process). ${ }^{37}$

Our study had several limitations. First, the use of self-reported measures to estimate sensory functions could potentially underestimate the association between famine exposure and sensory impairments, though this method has been widely adopted in epidemiological studies to assess visual and hearing functions. ${ }^{12} 13$ Second, using date of birth to define famine exposure might result in misclassification of exposed groups, but identifying an unexposed group not affected by famine was not feasible because the Chinese famine affected nearly the whole China. Finally, we used provincial EDR estimated from government reports as the proxy for famine severity, which may be subject to falsification, but EDR is still one of the most commonly used famine severity measures in China.

\section{CONCLUSIONS}

In conclusion, this national cohort study is among the first to estimate the long-term sensory function consequences of earlylife famine exposure in a non-European population, suggesting that exposure to famine during the gestation period and late childhood has everlasting adverse effects on hearing impairment and dual sensory impairments in adulthood. The study highlights the importance of providing sufficient nutrition during the critical earliest stages of development. 


\section{What is already known on this subject}

- Existing research suggests that early-life malnutrition pregnancy and infancy malnutrition played an important role in susceptibility to later non-communicable diseases .

- However, little is known about the association between severe nutritional deficiency during early-life and midlife sensory functions.

\section{What this study adds}

Severe nutritional deprivation during prenatal and postnatal periods has everlasting adverse effects on hearing and dual sensory impairments in Chinese adults in their early 50 s.

- This national cohort study highlights the importance of providing sufficient nutrition during the critical earliest stages of development to promote healthy ageing.

Acknowledgements The authors are grateful to the China Health and Retirement Longitudinal Study (CHARLS) team for providing data and simple training in using the database.

Contributors HF designed the study. HR and HF carried out the data analyses. All authors discussed the analysis results, wrote the manuscript, and read and approved the final version of the manuscript.

Funding This work was supported by the National Natural Science Foundation of China (grant number 71774006) — awarded to HF and China Postdoctoral Science Foundation (grant number 2019M650392) — awarded to HR.

Competing interests None declared.

Patient consent for publication Not required.

Provenance and peer review Not commissioned; externally peer reviewed.

Data availability statement Data are available in a public, open-access repository.

ORCID iD

Hai Fang http://orcid.org/0000-0003-0335-1685

\section{REFERENCES}

1 Bouscaren N, Yildiz H, Dartois L, et al. Decline in instrumental activities of daily living over 4-year: the association with hearing, visual and dual sensory impairments among non-institutionalized women. J Nutr Health Aging 2019;23:687-93.

2 Gopinath B, Liew G, Burlutsky G, et al. Visual and hearing impairment and retirement in older adults: a population-based cohort study. Maturitas 2017;100:77-81.

3 Golub JS, Brewster KK, Brickman AM, et al. Association of audiometric age-related hearing loss with depressive symptoms among hispanic individuals. JAMA Otolaryngol Head Neck Surg 2019;145:132-9.

4 Dawes P, Cruickshanks KJ, Moore DR, et al. The effect of prenatal and childhood development on hearing, vision and cognition in adulthood. PloS One 2015;10: e0136590.

5 Black RE, Victora CG, Walker SP, et al. Maternal and child undernutrition and overweight in low-income and middle-income countries. Lancet (London, England) 2013:382:427-51.

6 Smil V. China's great famine: 40 years later. BMJ (Clinical Research Ed) 1999:319:1619-21

7 Wang Z, Zou Z, Dong B, et al. Association between the great China famine exposure in early life and risk of arthritis in adulthood. J Epidemiol Community Health 2018;72:790-5.

8 Panza F, Quaranta N, Logroscino G. Sensory changes and the hearing loss-cognition link: the cognitive ear. JAMA Otolaryngol Head Neck Surg 2018;144:127-8.
9 Zhao Y, Hu Y, Smith JP, et al. Cohort profile: the China Health and Retirement Longitudinal Study (CHARLS). Int J Epidemiol 2014;43:61-8.

10 Luo H, Li J, Zhang Q, et al. Obesity and the onset of depressive symptoms among middle-aged and older adults in China: evidence from the CHARLS. BMC Public Health 2018;18:909.

11 Peng Y, Hai M, Li P, et al. Association of exposure to Chinese famine in early life with the risk of metabolic syndrome in adulthood. Ann Nutr Metab 2020;1-7. Epub 2020 Apr 7.

12 Maharani A, Dawes P, Nazroo J, et al. Visual and hearing impairments are associated with cognitive decline in older people. Age Ageing 2018;47:575-81.

13 Maharani A, Dawes P, Nazroo J, et al. Associations between self-reported sensory impairment and risk of cognitive decline and impairment in the Health and Retirement Study (HRS) cohort. I Gerontol B Psychol Sci Soc Sci 2019.

14 Andresen EM, Malmgren JA, Carter WB, et al. Screening for depression in well older adults: evaluation of a short form of the CES-D (Center for Epidemiologic Studies Depression Scale). Am J Prev Med 1994;10:77-84.

15 Suzuki K. The developing world of DOHaD. J Dev Orig Health Dis 2018;9:266-9.

16 Lesuis SL, Hoeijmakers L, Korosi A, et al. Vulnerability and resilience to Alzheimer's disease: early life conditions modulate neuropathology and determine cognitive reserve. Alzheimer's Res Ther 2018;10:95.

17 Xu H, Zhang Z, Li L, et al. Early life exposure to China's 1959-61 famine and midlife cognition. Int J Epidemiol 2018;47:109-20.

18 de Rooij SR, Mutsaerts H, Petr J, et al. Late-life brain perfusion after prenatal famine exposure. Neurobiol Aging 2019;82:1-9.

19 Rong H, Lai X, Mahmoudi E, et al. Early-life exposure to the Chinese famine and risk of cognitive decline. J Clin Med 2019;8:484.

20 Schubert CR, Cruickshanks KJ, Fischer ME, et al. Sensory impairments and cognitive function in middle-aged adults. I Gerontol A Biol Sci Med SCi 2017:72:1087-90.

21 Roseboom TJ. Epidemiological evidence for the developmental origins of health and disease: effects of prenatal undernutrition in humans. J Endocrinol 2019;242:T135-t44.

22 Franke K, Gaser C, Roseboom TJ, et al. Premature brain aging in humans exposed to maternal nutrient restriction during early gestation. Neuroimage 2018;173:460-71.

23 Rong $\mathrm{H}, \mathrm{Xi}$ Y, An Y, et al. The correlation between early stages of life exposed to chinese famine and cognitive decline in adulthood: nutrition of adulthood plays an important role in the link? Front Aging Neurosci 2017:9:444.

24 Roseboom T, de Rooij S, Painter R. The Dutch famine and its long-term consequences for adult health. Early Hum Dev 2006;82:485-91.

25 Verduci E, Martelli A, Miniello VL, et al. Nutrition in the first 1000 days and respiratory health: a descriptive review of the last five years' literature. Allergol Immunopathol (Madr) 2017;45:405-13.

26 Fukuoka H, Kubota T. One-carbon metabolism and lipid metabolism in DOHaD. Adv Exp Med Biol 2018;1012:3-9.

27 Kalm LM, Semba RD. They starved so that others be better fed: remembering Ancel Keys and the Minnesota experiment. J Nutr 2005;135:1347-52.

28 Pereira CF, Santos AE, Moreira PI, et al. Is Alzheimer's disease an inflammasomopathy? Ageing Res Rev 2019;100966.

29 Shen L, Li C, Wang Z, et al. Early-life exposure to severe famine is associated with higher methylation level in the IGF2 gene and higher total cholesterol in late adulthood: the Genomic Research of the Chinese Famine (GRECF) study. Clin Epigenetics 2019;11:88.

30 Penido AB, Rezende GH, Abreu RV, et al. Malnutrition during central nervous system growth and development impairs permanently the subcortical auditory pathway. Nutr Neurosci 2012;15:31-6.

$31 \mathrm{Li}$ J, Na L, Ma H, et al. Multigenerational effects of parental prenatal exposure to famine on adult offspring cognitive function. Sci Rep 2015;5:13792.

32 Gong R, Hu X, Gong C, et al. Hearing loss prevalence and risk factors among older adults in China. Int J Audiol 2018;57:354-9.

33 Zhu J, Xiang YB, Cai $\mathrm{H}$, et al. Associations of obesity and weight change with physical and mental impairments in elderly Chinese people. Maturitas 2018;108:77-83.

34 Kleinlogel S, Vogl C, Jeschke M, et al. Emerging approaches for restoration of hearing and vision. Physiol Rev 2020.

35 Heine C, Browning CJ, Gong CH. Sensory loss in China: prevalence, use of aids, and impacts on social participation. Front Public Health 2019;7:5.

36 Li JN, Chen S, Zhai L, et al. The advances in hearing rehabilitation and cochlear implants in China. Ear Hear 2017;38:647-52.

37 Li L, Lam J, Lu Y, et al. Attitudes of students, parents, and teachers toward glasses use in rural China. Arch Ophthalmol 2010;128:759-65. 